Preprint typeset in JHEP style - HYPER VERSION

DESY-03-140

ITP-Budapest-600

WUB-03-09

\title{
Pentaquark hadrons from lattice QCD
}

\author{
F. Csikor ${ }^{a}$, Z. Fodor ${ }^{a, b}$, S.D. Katz ${ }^{c *}$ and T.G. Kovács ${ }^{b \dagger}$ \\ ${ }^{a}$ Institute for Theoretical Physics, Eötvös University, Hungary \\ ${ }^{b}$ Department of Physics, University of Wuppertal, Germany \\ ${ }^{c}$ Deutsches Elektronen-Synchrotron, DESY Hamburg, Germany
}

\begin{abstract}
We study spin $1 / 2$ isoscalar and isovector candidates in both parity channels for the recently discovered $\Theta^{+}(1540)$ pentaquark particle in quenched lattice QCD. Our analysis takes into account all possible uncertainties, such as statistical, finite size and quenching errors when performing the chiral and continuum extrapolations and we have indications that our signal is separated from scattering states. The lowest mass that we find in the $I^{P}=0^{-}$channel is in complete agreement with the experimental value of the $\Theta^{+}$mass. On the other hand, the lowest mass state in the opposite parity $I^{P}=0^{+}$channel is much higher. Our findings suggests that the parity of the $\Theta^{+}$is negative.
\end{abstract}

KEYWORDS: at.

${ }^{*}$ On leave from Institute for Theoretical Physics, Eötvös University, Hungary

†On leave from Department of Theoretical Physics, University of Pécs, Hungary 


\section{Contents}

1. Introduction 1

2. Simulation details and data analysis 2

3. Results and conclusions 9

\section{Introduction}

The possible existence of exotic hadrons has long been put forward in several contexts, but the experimental discovery of the first such particle had to wait until early this year [1]-[ [4]. For a long time exotic hadron states containing more than three quarks were not considered to be of great importance on account of their presumed large decay widths. (For a recent discussion of this issue see e.g. [5].) The experimental discovery of the $\Theta^{+}(1540)$ particle changed this situation dramatically. Indeed, the tightest experimental upper bound so far on the width of the $\Theta^{+}$is around $10 \mathrm{MeV}$ [4], and based on the reanalysis of older experimental data, more stringent constraints on the width are also suggested [6]. This remarkably narrow width would explain why the $\Theta^{+}$has not been seen before.

Since the $\Theta^{+}$decays into a neutron and a $K^{+}$, its strangeness has to be +1 , the third component of its isospin is 0 , and its minimal quark content is dduus. From the lack of a signal in the $I_{3}=1$ channel the SAPHIR collaboration concluded that the $\Theta^{+}$should be an isospin singlet state [3]. Its spin and parity cannot be unambiguously pinned down based on currently available experimental data.

The experimental discovery of the $\Theta^{+}$pentaquark triggered a flurry of theoretical speculations [7] about its possible structure, yet unmeasured quantum numbers and on the possibility of the existence of other exotic hadrons. Chiral soliton models [B], as well as correlated quark models [9, 10, 11] suggest that the $\Theta^{+}$should be a spin $1 / 2$ positive parity isosinglet state. In contrast, uncorrelated quark models predict negative parity. QCD sum rules have also been used to study the $\Theta^{+}[12]$.

In the present work we use lattice QCD to study the properties of some pentaquark states that are likely candidates to be identified with the $\Theta^{+}$. This is the only available tool to extract low energy hadronic properties starting from first principles, i.e. QCD. In our exploratory study we choose to work in the quenched approximation, which is known to be successful in reproducing mass ratios of stable hadrons $[13]-[16]$. 
Working at three different values of the lattice spacing, $a=0.17,0.12$ and $0.09 \mathrm{fm}$ enables us to do a continuum extrapolation. Since using light enough quarks that reproduce the physical pion mass would be prohibitively costly, for each lattice spacing we simulate at four different, somewhat larger pion masses and extrapolate to the physical limit. This is done in two different ways, by keeping the $s$-quark mass fixed to reproduce the physical kaon mass in the chiral limit and also by changing the $s$-quark mass in a way to keep the nucleon to kaon mass ratio fixed as the light quark mass is varied. In order to have finite size effects under control we always keep the linear physical size of the spatial box fixed at $L \approx 2 \mathrm{fm}$. In addition, on the two coarsest lattices, we also perform a finite volume analysis by considering different volumes.

Since the exact structure of the $\Theta^{+}$is yet unknown, there are quite a few different possibilities to construct a baryon with the given quark content $d d u u \bar{s}$. In our computation we use two particular spin $1 / 2$ operators, one with isospin $I=0$ and and another with $I=1$. The analysis is carried out in both parity channels.

The main result of the present work is that the lowest mass that we find in the $I^{P}=0^{-}$channel is in complete agreement with the experimental value of the $\Theta^{+}$ mass. On the other hand, the lowest mass in the opposite parity $I^{P}=0^{+}$channel turns out to be much larger, thus we suggest negative parity for the $\Theta^{+}$particle.

The lightest state in the $I=1$ channel also has negative parity and comes out to be about $15 \%$ heavier, but still within one $\sigma$ of the $\Theta^{+}$mass. The SAPHIR Collaboration, however, have already ruled out the $\Theta^{+}$to be a member of an $I>0$ multiplet [3], therefore the triplet state that we found might be a genuinely new state not seen before, albeit with a potentially much larger width than that of the $\Theta^{+}$.

The rest of the paper is organized as follows. In Section 2 we give the details of our simulations and our data analysis. In Section 3 we present our results and conclusions. Those who are not interested in lattice technicalities can directly skip to Section 3 .

\section{Simulation details and data analysis}

In this section we discuss some technical details of our lattice simulations. We use the Wilson gauge action and the Wilson fermion action. The only "non-standard" piece of the calculation is the choice of the pentaquark operator. Since there is general agreement in the literature that the $\Theta^{+}$is very likely to have spin $1 / 2$, we restricted ourselves to this choice. Nevertheless, it will be interesting to look at higher spin states too. After fixing the quark content of the particle to be $d d u u \bar{s}$ and its spin to $1 / 2$, there is still considerable freedom in choosing the interpolating operator. Even though the $\Theta^{+}$is experimentally suggested to be an isosinglet, we computed 
correlators both in the $I=0$ and $I=1$ channels using the interpolating fields

$$
\eta_{0 / 1}=\epsilon^{a b c}\left[u_{a}^{T} C \gamma_{5} d_{b}\right]\left\{u_{e} \bar{s}_{e} i \gamma_{5} d_{c} \mp(u \leftrightarrow d)\right\} .
$$

Here $C$ is the charge conjugation operator, only colour indices are explicitly written out and the $I=0$ case corresponds to the minus sign. There are many more possibilities even in the $\mathrm{I}=0,1$ channels. In principle testing other interpolating operators and checking which one has the best overlap with the $\Theta^{+}$state should provide information on the wave function of the particle. In this exploratory study, however, we do not pursue this direction any further.

We did not study the $I=2$ channel either, mainly because it seems to be computationally more expensive. At first sight this is hard to understand since normally in lattice simulations one is used to quark propagator computations being the most expensive part. Given the propagators, calculating the different contractions entering hadron correlators takes only a tiny fraction of the time. With these more complicated pentaquark hadrons, however, that is no longer the case. Already in the $I=0,1$ channels the time for computing the contractions is of the same order of magnitude as the fermion matrix inversion. The reason for that is mainly that the number of terms occurring in the colour and Dirac index sums grows exponentially with the number of constituent particles.

The $\Theta^{+}$can decay into a kaon and a nucleon even in the quenched approximation. The experimentally very narrow pentaquark state is just slightly above the $N+K$ threshold, thus one expects that the $\Theta^{+}$is visible in correlators of our well localised operators. Note that in principle scattering states can mix in the spectrum, though they have a characteristic volume dependence. (This volume dependence can be used to calculate decay rates c.f. [17]). On

\begin{tabular}{|l||r|r|r|r|}
\hline$\beta$ & size & $a(\mathrm{fm})$ & $\mathrm{L}(\mathrm{fm})$ & confs \\
\hline \hline 5.70 & $10^{3} \times 24$ & 0.171 & 1.71 & 200 \\
\hline 5.70 & $12^{3} \times 24$ & 0.171 & 2.05 & 220 \\
\hline 5.70 & $16^{3} \times 32$ & 0.171 & 2.73 & 100 \\
\hline 5.85 & $10^{3} \times 32$ & 0.123 & 1.23 & 726 \\
\hline 5.85 & $12^{3} \times 32$ & 0.123 & 1.48 & 348 \\
\hline 5.85 & $16^{3} \times 32$ & 0.123 & 1.97 & 90 \\
\hline 6.00 & $20^{3} \times 36$ & 0.093 & 1.86 & 94 \\
\hline
\end{tabular}

Table 1: The seven gauge field ensembles used for the study of the pentaquark. The lattice spacing has been set with the Sommer parameter $r_{0}=0.50 \mathrm{fm}$.

the two coarsest lattices we searched for any possible volume dependence of the $\Theta^{+}$by considering different spatial lattice sizes ranging from $L=1.3$ to $2.7 \mathrm{fm}$. We did not see the expected volume dependence characteristic to the S-wave scattering state. (Note, however that this signature would appear only in the $I=1$ channel, since in the $I=0$ channel the scattering length is too small.) In addition, on our largest lattice at the largest quark mass, based on 300 configurations, we computed the $2 \times 2$ correlation matrix of the $\eta$ operator of Eq. (2.1) and a similar operator with its colour indices contracted as in the nucleon and the kaon. The latter is expected to couple more strongly to the 
scattering state. After analysing the cross correlator matrix we obtained for the smallest energy state $E_{0} /\left(m_{N}+m_{K}\right)=0.994(18)$ (which we interpret as the S-wave scattering state) and for the state above $E_{1} /\left(m_{N}+m_{K}\right)=1.074(20)$ (which is the $\Theta^{+}$candidate). Note that the volume of our spatial box implies that the next scattering state with zero total momentum should not occur below $E /\left(m_{N}+m_{K}\right)=1.21$. Thus, in particular, we do not expect to be faced with the unlucky scenario that in our analysis one of the scattering states is accidentally lifted to the $\Theta^{+}$mass.

Given our choice of interpolating operators for the pentaquark, we now discuss other details of the simulations. We generated seven different ensembles of quenched gauge field configurations with the Wilson plaquette action. Quark propagators were calculated using Wilson fermions. The parameters of our gauge ensembles are summarized in Table 1. Ensembles with three different values of the coupling were chosen to have roughly the same spatial volume in order to avoid any finite size contamination.

The last ingredients of our computation are the choice of quark masses and the way we fix the two independent parameters, the lattice spacing and the $s$-quark mass. Due to the extensive literature on quenched spectroscopy one can safely plan the necessary parameter sets in order to carry out acceptable chiral and continuum extrapolations. We used light quark masses in the range $m_{\pi} / m_{\rho}=0.5-0.7$. In quenched QCD there is a small inherent ambiguity in how those parameters can be fixed. Given the fact that the $\Theta^{+}$is intimately connected to the kaon, it seems reasonable to use the physical mass of the kaon to set the $s$-quark mass. We adopted two different schemes in approaching the chiral limit. In both cases the light quarks were taken degenerate. In scheme (A) we kept the $s$-quark mass fixed to reproduce the physical kaon mass in the chiral limit. In scheme (B) we changed the mass of the $s$-quark along with that of the light quarks so as to keep the nucleon to kaon mass ratio constant.

One of the main questions we set out to address in the present study is to determine the parity of the $\Theta^{+}$particle. Simple baryon interpolating operators in general are expected to couple to states of both parities [18, 19]. With the parity assignment of our operators the correlators are of the form ${ }^{1}$

$$
\left\langle B_{0} \bar{B}_{t}\right\rangle=\left(1-\gamma_{0}\right)\left[C_{+} \mathrm{e}^{-t m_{+}}+C_{-} b \mathrm{e}^{-(T-t) m_{-}}\right]+\left(1+\gamma_{0}\right)\left[C_{+} b \mathrm{e}^{-(T-t) m_{+}}+C_{-} \mathrm{e}^{-t m_{-}}\right],
$$

where $T$ is the size of the lattice in the time direction, $b= \pm 1$ corresponds to periodic/antiperiodic boundary conditions in the time direction, and $m_{ \pm}$are the masses of the \pm parity states. To be able to project out the two parities separately,

\footnotetext{
${ }^{1}$ In the first version of this paper the signs of the terms proportional to $\gamma_{0}$ were opposite. We thank S. Sasaki 20] and K.F. Liu 21 for pointing out this apparent error. The only consequence of correcting this error is the flip of the parity assignment. All the other results, including correlators and masses remained unchanged.
} 
we always computed both components of the correlators, i.e. the one proportional to 1 and $\gamma_{0}$. In addition, at one quark mass on each ensemble we also computed the correlators with antiperiodic boundary condition, which enabled us to determine the two parity partners in another way.

We performed two-parameter correlated fits to the 1 and $\gamma_{0}$ components of the $\left\langle B_{0} \bar{B}_{t}\right\rangle$ pentaquark correlators with the standard cosh and sinh form respectively, assuming that at large enough separation the lightest state dominates the correlators. Since our data sample was rather limited we followed Ref. [22] and used correlated fitting with smeared smallest eigenvalues of the correlation matrix. We could always find fit ranges with acceptable $\chi^{2} \approx 1$ accompanied by a plateau in the effective and in the fitted masses. The fits to the 1 and $\gamma_{0}$ components always gave compatible results. The interpolating operators, however, could be better chosen, since the mass plateaus were reached at relatively large time separations compared to e.g. the nucleon correlator. In Fig. 11 we show a typical set of fits in the $I=0$ channel.

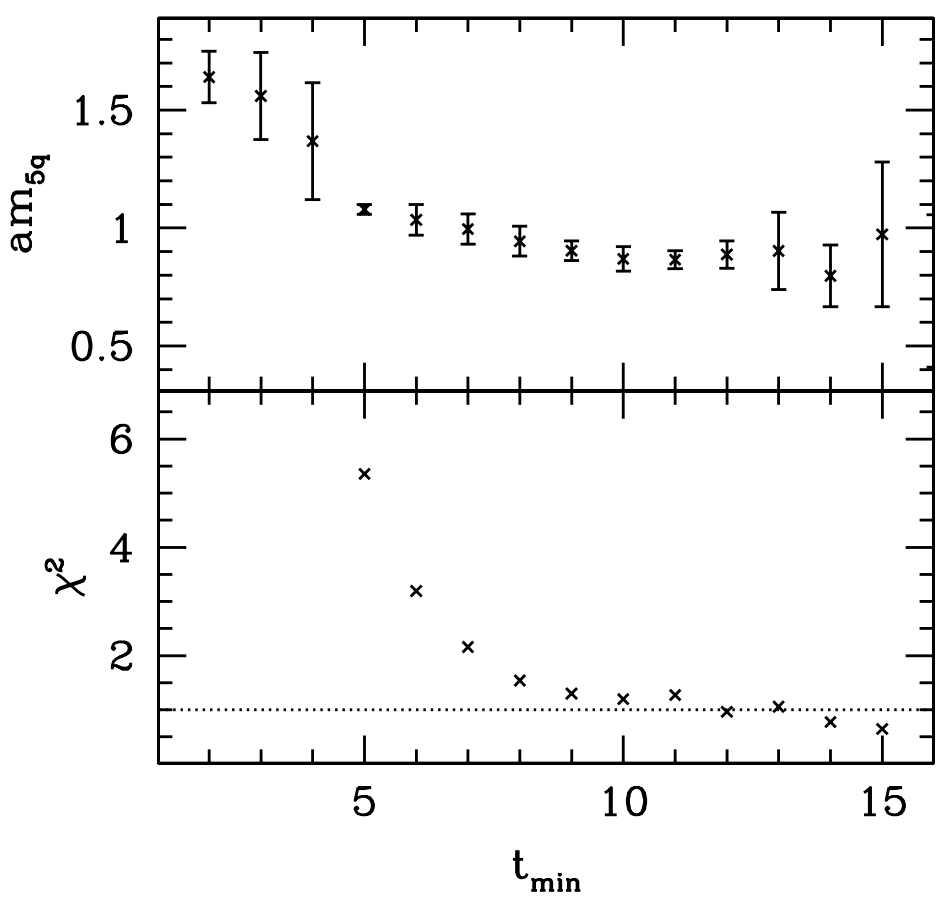

Figure 1: A typical fit for a correlator in the $I=0$ channel at $\beta=5.85$ at our lightest $u$ quark. The mass given by the fit along with the corresponding $\chi^{2}$ per degree of freedom is shown versus the starting point of the fit range.

Since one of our most important goals is to determine the parity, let us discuss this issue in some detail. The interpolating operators we used are expected to couple to states of both parities in the given channel. In order to determine the parity of the lowest state as well as the mass of its heavier parity partner we performed single 
exponential fits to both the $1+\gamma_{0}$ and the $1-\gamma_{0}$ components of the correlators. As can be seen from Eq. (2.2) these projections should fall off with two different exponents for $0 \leq t \ll T / 2$ and for $T / 2 \ll t<T$, corresponding to the two different parity states. As expected, we could always confirm that one of the exponents was compatible with our previously found lowest mass obtained by the cosh and sinh fits to the 1 and $\gamma_{0}$ components, respectively. Both for the $I=0$ and the $I=1$ channels, the negative parity state turned out to be the lighter one of the two parities.

The above correlators were calculated with periodic quark boundary conditions. An independent method to determine the parity is based on the combination of the correlation functions obtained with periodic and antiperiodic boundary conditions. We carried out this analysis at each lattice spacing for the largest quark masses. As can be seen from Eq. (2.2), the sum (difference) of the 1 component of the periodic and the $\gamma_{0}$ component of the antiperiodic correlator projects onto the negative (positive) parity state, respectively. Single mass fits to these combinations yielded masses in complete agreement with our mass and parity determinations using periodic boundary conditions.

A consistent picture emerged for the parity of the pentaquark states at all lattice spacings and quark masses. The negative parity mass was always by many standard deviations below the positive parity mass. This result was obtained from both boundary conditions combining the 1 and $\gamma_{0}$ channels and also from combining different boundary conditions. Thus, we conclude that both for the $I=0$ and the $I=1$ channels, the negative parity state was determined to be the lighter one of the two parities.

The masses in the $I^{P}=0^{ \pm}, 1^{ \pm}$channels were then extrapolated to the chiral limit. As we already mentioned, for the chiral extrapolations we used two different sets of quark masses and correspondingly two different methods.

(A) We set the scale by the Sommer parameter $r_{0}=0.5 \mathrm{fm}$ and we held the $s$ quark mass fixed to reproduce the physical kaon mass $m_{K}=494 \mathrm{MeV}$. This approach resulted in strange quark hopping parameters of $\kappa_{s}=0.1621,0.1574$ and 0.1544 for $\beta=5.70,5.85$ and 6.00 , respectively. At all three lattice spacings four different light quark masses were used in order to extrapolate into the chiral limit.

(B) A somewhat different method to approach the chiral and continuum limit can be imagined as follows. For all the three lattice spacings we used four sets of strange and light hopping parameters. The light quark masses were used to extrapolate into the chiral limit, whereas we chose the strange quark mass to reproduce the experimental nucleon to kaon mass ratio.

We followed both methods all the way through, first performing the chiral extrapolation at fixed $\beta$ then carrying out the continuum extrapolation. The two methods 
(A and B) should give identical final results, though the difficulties connected with the chiral and continuum extrapolations might be quite different. Luckily enough, the extrapolations for the relevant mass ratios were equally easy for both techniques. The obtained results are in complete agreement. Since we used the same gauge configuration ensembles for the two methods, the results are strongly correlated and cannot be combined. In what follows, we focus on the somewhat more conventional method (A).

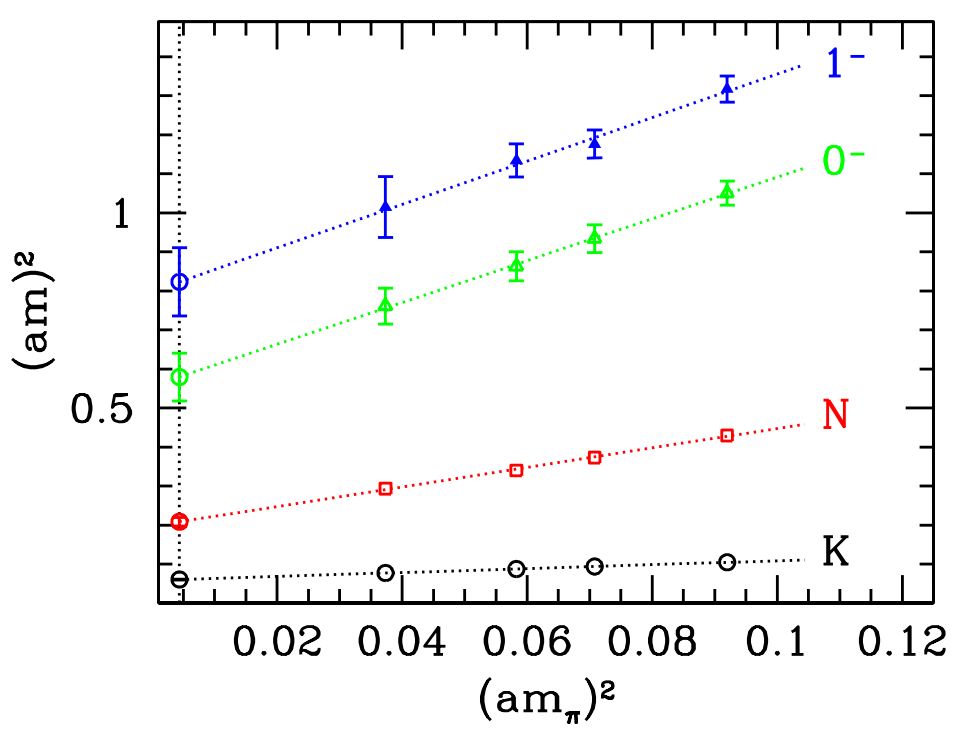

Figure 2: A typical chiral extrapolation of hadron masses on our finest lattice at $\beta=6.0$. The particles are (from top to bottom) the $I=1,0$ negative parity pentaquark states, the nucleon and the kaon.

The chiral extrapolations for hadron masses can be performed with the fit functions

$$
m_{H}^{2}=a+b m_{\pi}^{2} \quad \text { or } \quad m_{H}^{2}=a+b m_{\pi}^{2}+c m_{\pi}^{3} .
$$

The difference between the two extrapolations gives some information about systematic uncertainties in the extrapolated quantities. Note, however, that our quark masses are quite small and we have only four different quark masses at each $\beta$. Thus with our statistics the quadratic fit turned out to be appropriate. Comparing our spectrum with similar results in the literature (c.f. [14]) we concluded that the uncertainties due to the chiral logarithms in the physical limit were subdominant at our present statistics. A typical chiral extrapolation for the kaon, the nucleon and the negative parity $I=0,1$ pentaquark states is shown in Fig. 2. Note, that our 
chirally extrapolated masses are merely illustrations. Since quenched spectroscopy is quite reliable for mass ratios of stable particles, it is physically even more motivated to extrapolate mass ratios instead of masses (or equivalently one might use the squared ratios suggested by the above mass fit formula). As we will see the quark mass dependence of these ratios can be weaker than that of the individual hadron masses.

The pentaquark states have the same quark content as the nucleon and the kaon. Therefore, a particularly attractive dimensionless quantity to look at is the mass ratio of a pentaquark state to the nucleon plus kaon mass. We also did chiral extrapolations of this mass ratio squared, and an example of that for both isospins and parities is shown in Fig. 3 .

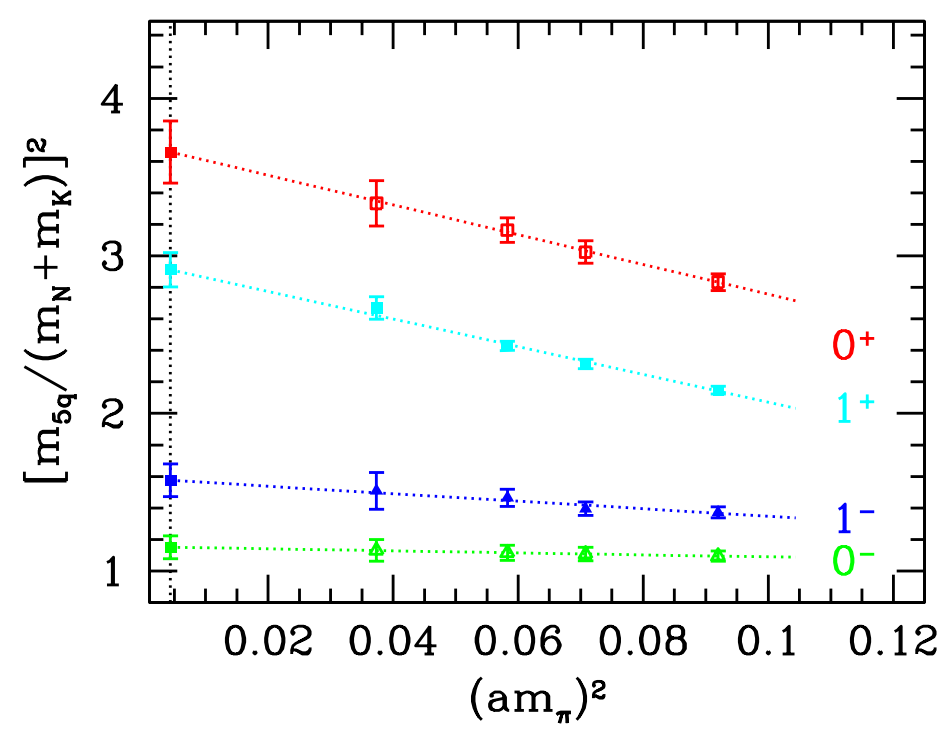

Figure 3: Chiral extrapolation of the squared mass ratios $m_{5 \mathrm{q}}^{2} /\left(m_{N}+m_{K}\right)^{2}$ on our finest lattice at $\beta=6.00$.

Finally, we performed a continuum extrapolation for the chirally extrapolated quantities. In Fig. 1 we demonstrate the continuum extrapolation of the pentaquark to nucleon plus kaon mass ratio for all the four pentaquark states. Since in general the Wilson action is known to have $\mathcal{O}(a)$ discretization errors, we extrapolated linearly to $a \rightarrow 0$. The mass ratios at hand show remarkably small scaling violations. 


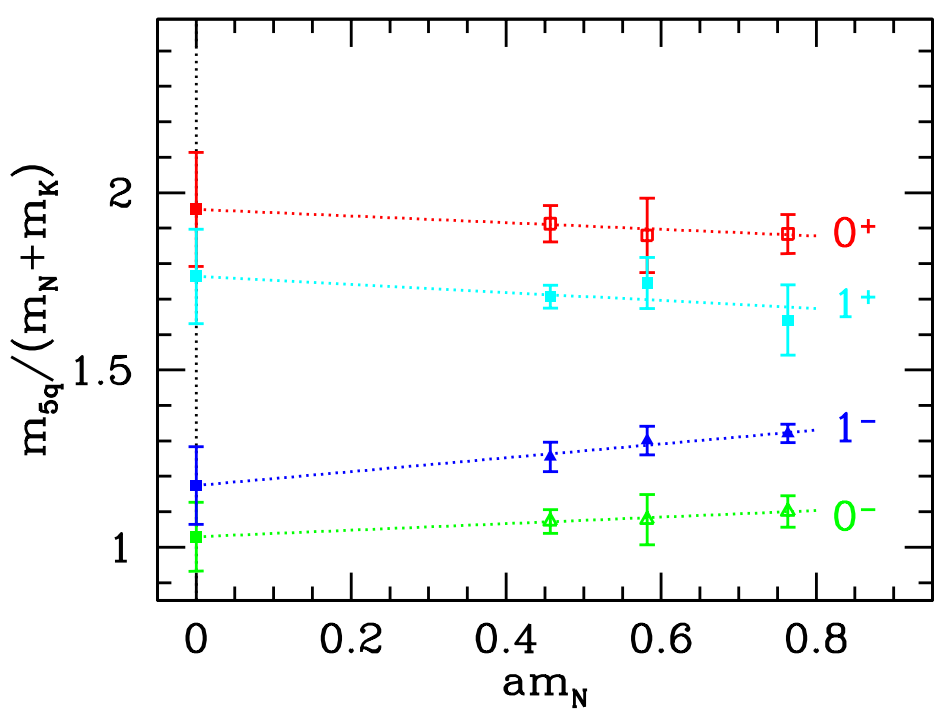

Figure 4: Continuum extrapolation of the mass ratios $m_{5 \mathrm{q}} /\left(m_{N}+m_{K}\right)$ for the various pentaquark states. The horizontal axis shows the nucleon mass in lattice units. The lines are linear fits to the data.

\section{Results and conclusions}

Before we present our final results we discuss how we handled the possible sources of errors. In lattice QCD there are five typical sources of uncertainties: $i$. statistical; $i i$. finite size, $i i i$. quark mass, $i v$. finite lattice spacing and $v$. quenching errors. We had all of these five error sources under control in our analysis.

$a d i$. Statistical uncertainties are well understood and they were included by standard techniques. These techniques need uncorrelated configurations, which we ensured by separating the analyzed configurations by as much as 1000 sweeps. Statistical errors were always estimated using a full jackknife analysis. The statistical uncertainties of our pentaquark masses are typically on the few percent level.

ad ii. Finite size effects in our quenched analysis turned out to be negligible. Our spatial box size was around $2 \mathrm{fm}$. In order to look for finite size corrections we also performed calculations on our two coarsest lattices using different box sizes. With our statistical uncertainties of order a few percent we could not find any size dependence in the hadron masses.

ad iii. Quark masses in lattice spectroscopy are usually treated differently in the strange and in the light (up or down) sectors. It is straightforward to take the physical strange quark mass. Due to computational/technical difficulties most of 
the analyses use extrapolations from light quark masses, which are larger than the physical one. We also applied this method. We included all the extrapolation errors into our analysis.

$a d i v$. Finite lattice spacing is an inherent source of uncertainty in any lattice analysis. We used the standard approach. We performed our calculations at three different lattice spacings and extrapolated to the continuum limit. Luckily enough, the mass ratios $m_{5 q} /\left(m_{N}+m_{K}\right)$ have a rather weak dependence on the lattice spacing (c.f. Fig. (4). Nevertheless, the quite large, order $10 \%$ errors on the finally quoted values are mostly due to the chiral and the continuum extrapolation.

$a d$. $v$. We used quenched gauge configurations, which is another source of systematic errors. Note, however, that in the case of stable hadrons, this is not expected to be very important. Indeed, as can be seen from Refs. [13] with an appropriate definition of the scale, the mass ratios of stable hadrons are described correctly by the quenched approximation on the 1-2\% level. We included a conservative estimate of $3 \%$ quenching uncertainty in our analysis.

In addition to these five typical sources of uncertainty, there is a somewhat special one, connected to the fact that the $\Theta^{+}$mass is rather close to the $\mathrm{N}+\mathrm{K}$ threshold. In the negative parity channel the $\mathrm{S}$-wave scattering state might contaminate the spectrum. Though we showed how these states can be successfully separated, more work is needed to clarify this issue and finalise the conclusion on the parity.

Given the fact that in the $m_{0^{-}} /\left(m_{K}+m_{N}\right)$ mass ratio we do not observe any scaling violations, we could also quote the value of this quantity on our finest lattice, which has the smallest error. This would give

$$
\frac{m_{0^{-}}}{m_{K}+m_{N}}=1.073(34),
$$

or using the physical kaon and nucleon mass

$$
m_{0^{-}}=1539 \pm 50 \mathrm{MeV}
$$

Note, that the continuum extrapolation increases the error on the mass ratio, and consequently on the mass, by a factor of 3 .

Our final result after continuum extrapolation is shown in Fig. 5. Of the four $I^{P}$ channels, the closest in mass to the experimentally observed $\Theta^{+}$is the $0^{-}$state. The $1^{-}$state can be seen to be about $15 \%$ heavier, but still within one standard deviation of the $\Theta^{+}$mass. This state, however, which is a member of an isospin triplet, is not the $\Theta^{+}$, since the latter is experimentally known to be an isospin singlet [3]. The parity partner of the $0^{-}$state is almost two times heavier and lies several standard deviations above the experimentally observed mass of the $\Theta^{+}$. This suggests that the $0^{-}$state should be identified with the $\Theta^{+}$.

Obviously in this exploratory study we could not perform a high statistics analysis. Quenching effects are quite small, nevertheless, it would be advisable to carry 


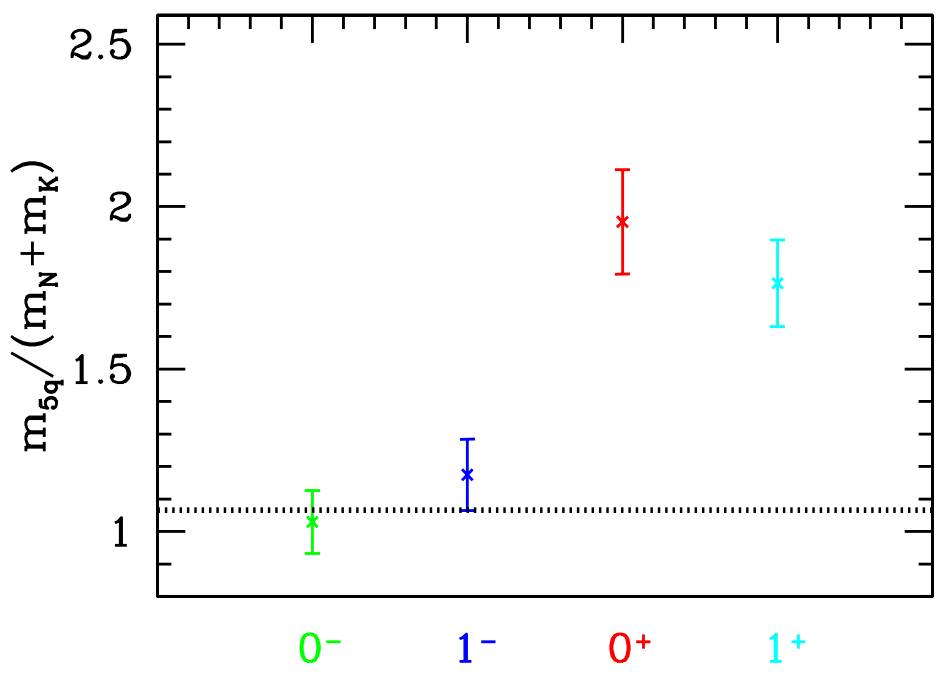

Figure 5: The continuum extrapolated mass ratios $m_{5 \mathrm{q}} /\left(m_{N}+m_{K}\right)$ for the lowest mass pentaquark states in the various $I^{P}$ channels. The horizontal line shows the experimentally known mass of the $\Theta^{+}$.

out the calculations with unquenched configurations. We did not make a systematic study of the possible interpolating operators that are likely to have a good overlap with the $\Theta^{+}$, either. This study would be also important. Comparing different spatial and flavour trial wave functions is practically the only way to obtain more information about the flavour structure of the $\Theta^{+}$wave function and decide among the several possibilities that have already been put forward in the recent literature (e.g. one could analyze diquark-diquark-antiquark pictures [9]). Furthermore, one expects additional exotic pentaquark hadrons with different spin, isospin and strangeness quantum numbers. Lattice QCD might be useful to test these light exotic particle cascades numerically.

In conclusion, we studied spin $1 / 2$ isoscalar and isovector candidates in both parity channels for the recently discovered $\Theta^{+}(1540)$ pentaquark particle in quenched lattice QCD. Our analysis took into account all possible uncertainties. The lowest mass that we find in the $I^{P}=0^{-}$channel is in complete agreement with the experimental value of the $\Theta^{+}$mass. On the other hand, the lowest mass state in the opposite parity $I^{P}=0^{+}$channel is much higher. Since the $I=1$ channel has already been excluded by experiment as a candidate for the $\Theta^{+}$[3], this leaves the $I^{P}=0^{-}$ state to be identified with the $\Theta^{+}$. Our conclusion is consistent with that of the in- 
dependent analysis of S. Sasaki [24], who separated in addition also the $\mathrm{K}+\mathrm{N}$ S-wave scattering state by observing a second plateau in the effective mass. This successful separation is extremely important for establishing the parity of the $\Theta^{+}$. Clearly, the

more sophisticated, CPU expensive, cross correlator technique is needed in order to unambigously separate the $\Theta^{+}$and the $\mathrm{K}+\mathrm{N}$ S-wave and its excited states, which will finalize the conclusion on the mass and parity.

\section{Acknowledgments}

We would like to thank K.-F. Liu, I. Montvay, F. Niedermayer and S. Sasaki for useful discussions. This research was partially supported by OTKA Hungarian Science Grants No. T34980, T37615, M37071, T032501. The computations were carried out at the Eötvös University on the 163 node PC cluster of the Department for Theoretical Physics, using a modified version of the publicly available MILC code (see www.physics.indiana.edu/sg/milc.html). T.G.K. also acknowledges support by a Bolyai Fellowship.

\section{References}

[1] T. Nakano et al., Phys. Rev. Lett. 91, 012002 (2003).

[2] S. Stepanyan et al. [CLAS Collaboration], arXiv:hep-ex/0307018.

[3] J. Barth et al. [SAPHIR Collaboration], arXiv:hep-ex/0307083.

[4] V. V. Barmin et al. [DIANA Collaboration], arXiv:hep-ex/0304040.

[5] R. W. Gothe and S. Nussinov, arXiv:hep-ph/0308230.

[6] S. Nussinov, arXiv:hep-ph/0307357; R. A. Arndt, I. I. Strakovsky and R. L. Workman, arXiv:nucl-th/0308012.

[7] S. Capstick, P. R. Page and W. Roberts, Phys. Lett. B 570, 185 (2003) [arXiv:hep-ph/0307019]; J. Randrup, arXiv:nucl-th/0307042; A. Hosaka, arXiv:hep-ph/0307232; T. Hyodo, A. Hosaka and E. Oset, arXiv:nucl-th/0307105;

X. Chen, Y. Mao and B. Q. Ma, arXiv:hep-ph/0307381; L. W. Chen, V. Greco, C. M. Ko, S. H. Lee and W. Liu, arXiv:nucl-th/0308006; P. Bicudo and G. M. Marques, arXiv:hep-ph/0308073; W. Liu and C. M. Ko, arXiv:nucl-th/0308034; S. I. Nam, A. Hosaka and H. C. Kim, arXiv:hep-ph/0308313; B. Jennings and K. Maltman, arXiv:hep-ph/0308286; B. G. Wybourne, arXiv:hep-ph/0307170.

[8] D. Diakonov, V. Petrov and M. V. Polyakov, Z. Phys. A 359, 305 (1997) [arXiv:hep-ph/9703373]; M. Praszalowicz, arXiv:hep-ph/0308114; M. V. Polyakov and A. Rathke, arXiv:hep-ph/0303138; H. Walliser and V. B. Kopeliovich, 
arXiv:hep-ph/0304058. D. Borisyuk, M. Faber and A. Kobushkin, arXiv:hep-ph/0307370; H. C. Kim, arXiv:hep-ph/0308242.

[9] R. L. Jaffe and F. Wilczek, arXiv:hep-ph/0307341;

[10] K. Cheung, arXiv:hep-ph/0308176; R. D. Matheus, F. S. Navarra, M. Nielsen, R. R. da Silva and S. H. Lee, arXiv:hep-ph/0309001.

[11] F. Stancu and D. O. Riska, arXiv:hep-ph/0307010; M. Karliner and H. J. Lipkin, arXiv:hep-ph/0307243; L. Y. Glozman, arXiv:hep-ph/0308232.

[12] S. L. Zhu, arXiv:hep-ph/0307345; C. E. Carlson, C. D. Carone, H. J. Kwee and V. Nazaryan, arXiv:hep-ph/0307396.

[13] C. Gattringer et al. [BGR Collaboration], arXiv:hep-lat/0307013.

[14] S. Aoki et al. [CP-PACS Collaboration], Phys. Rev. D 67, 034503 (2003) [arXiv:hep-lat/0206009].

[15] C. R. Allton, V. Gimenez, L. Giusti and F. Rapuano, Nucl. Phys. B 489, 427 (1997) [arXiv:hep-lat/9611021].

[16] H. P. Shanahan et al. [UKQCD Collaboration], Phys. Rev. D 55, 1548 (1997) [arXiv:hep-lat/9608063].

[17] L. Lellouch and M. Luscher, Commun. Math. Phys. 219 (2001) 31 [arXiv:hep-lat/0003023].

[18] I. Montvay and G. Munster, "Quantum Fields On A Lattice," Cambridge University Press, 1997.

[19] S. Sasaki, T. Blum and S. Ohta, Phys. Rev. D 65, 074503 (2002) [arXiv:hep-lat/0102010].

[20] S. Sasaki, private communication of Sep. 16. 2003.

[21] K.F. Liu, private communication of Sep. 16. 2003.

[22] C. Michael and A. McKerrell, Phys. Rev. D 51, 3745 (1995) [arXiv:hep-lat/9412087].

[23] M. Gockeler, R. Horsley, H. Perlt, P. Rakow, G. Schierholz, A. Schiller and P. Stephenson, Phys. Rev. D 57, 5562 (1998) [arXiv:hep-lat/9707021].

[24] S. Sasaki, arXiv:hep-lat/0310014. 